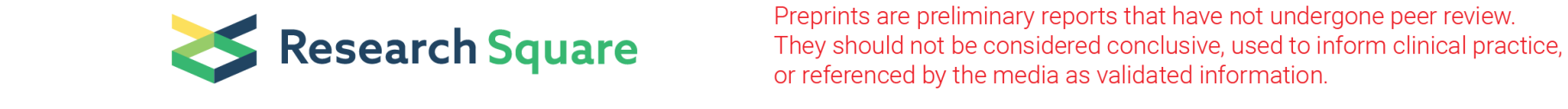

\title{
Predicting The Sign of Trophic Effects: Individual-Based Simulation Versus Loop Analysis
}

Virág Adrienn Fábián ( $\square$ fabian.virag.adrienn@gmail.com )

Centre for Ecological Research

Ferenc Jordán

Central European University

\section{Research Article}

Keywords: food web, effect sign, loop analysis, individual-based simulation, systems-based conservation

Posted Date: May 20th, 2021

DOI: https://doi.org/10.21203/rs.3.rs-524228/v1

License: () (i) This work is licensed under a Creative Commons Attribution 4.0 International License. Read Full License

Version of Record: A version of this preprint was published at Community Ecology on December 2nd, 2021. See the published version at https://doi.org/10.1007/s42974-021-00068-1. 


\section{Abstract}

Food web research needs to be predictive in order to support decisions system-based conservation. In order to increase predictability and applicability, complexity needs to be reduced to simple and clear results. One question emerging frequently is whether certain perturbations (environmental effects or human impact) have positive or negative effects on natural ecosystems or their particular components. Yet, most of food web studies do not consider the sign of effects.

Here, we study 6 versions of the Kelian River (Borneo) food web, representing six study sites along the river. For each network, we study the sign of the effect of a perturbed trophic group $i$ on each other $j$ groups. We compare the outcome of the relatively complicated dynamical simulation model and the relatively simple loop analysis model. We compare these results for the 6 sites and also the 14 trophic groups. Finally, we see if sign-agreement and sign-determinacy depend on certain structural features (node centrality, interaction strength).

We found major differences between different modelling scenarios, with herbivore-detritivore fish behaving in the most consistent, while algae and particulate organic matter behaving in the least consistent way.

\section{Introduction}

In complex, multi-species ecosystems, a number of interactions connect various organisms. Predicting the community-wide effects of single-species perturbations is a challenge for both ecological research and systems-based management.

In complex ecological systems, the multiplicity of direct and indirect interactions make it uneasy to provide simple and clear predictions on the effect of single-node perturbations. The effects on other organisms and, generally, community response is the outcome of a number of interconnected pathways. Predicting whether the influence of organism $i$ on organism $j$ will be positive or negative is not easy, even without considering non-trophic effects and complicated functional responses. Also the experimental results on positive inter-specific interactions are quite recent $[1,2,3]$. Considering effect sign is critically important, for example, if positive feedback loops $[4,5]$ or mutualisms $[6]$ are to be understood.

Network analysis offers various methods for predicting effect sign. Loop analysis [7, 8], mixed trophic impact $[9,10]$, signed topological importance $[11,12]$ and dynamical simulations [13] can be used but their relationship is not straightforward (i.e. whether they provide similar or complementary information). Yet, loop analysis has been used extensively together with topological network analysis (e.g.[14, 15, 16]).

Generally speaking, topological models provide fast and easy but not very realistic results from a quite static viewpoint. Sophisticated food web simulations provide more accurate results but need a lot of data and offer results that can be harder to interpret. Qualitative models, like loop analysis, are somewhere in between, trying to combine simplicity (without explicit dynamics) and reality (interaction sign considered).

In this paper, we study food webs at the level of individual nodes (trophic groups), interspecific interactions and whole networks. We compare (1) the results of loop analysis and dynamical simulations predicting the sign of effects following single-node perturbations. Beyond comparing these two methods themselves, we also compare (2) the 6 food web models as well as (3) the 14 trophic groups. Finally, (4) we ivestigate if there is an correlation between various structural properties and sign-agreement.

\section{Data And Methods}

\section{Data}

We used 6 versions of the Kelian River (Borneo) food web for our analysis (data from [17, 18]). This makes it possible to assess spatial variability within the ecosystem.

The food web is described for 6 different locations along the river $[13,19]$, based on extensive earlier field work [17, 18$]$. The 6 sites represent a gradient from a pristine rainforest to a human settlement. The pristine food web (site 1) contains 14 trophic groups and 2 additional groups appear only downstream, so the total number of trophic groups is 16 (see Table 1). Most of the groups represent living organisms (e.g. PRED: invertebrate predators) but there are some non-living groups as well (e.g. POM: settled and suspended 
coarse and fine Particulate Organic Matter). The defintion of the groups and the description of interactions among them are based on long-term, extensive field work $[17,18]$.

Table 1. The abbreviation for the 16 trophic groups composing the 6 food webs.

\begin{tabular}{|ll|}
\hline ALGA & green and blue-green algae \\
\hline CARN & carnivorous fish \\
\hline COLF & invertebrate collector-filterers \\
\hline COLG & invertebrate collector-gatherers \\
\hline DIAT & diatoms \\
\hline FILA & filamentous bacteria \\
\hline GRAZ & invertebrate grazers \\
\hline HEDE & herbivore-detritivore fish \\
\hline HERB & herbivorous fish \\
\hline HUMW & human waste \\
\hline LEAF & leaf litter \\
\hline OMNI & omnivorous fish \\
\hline POM & settled and suspended coarse and fine Particulate Organic Matter \\
\hline PRED & invertebrate predators \\
\hline SHRE & invertebrate shredders \\
\hline TERR & terrestrial insects \\
\hline
\end{tabular}

Food web structure: topological importance

We quantified the topology of the food webs based on the topological importance $\left(\mathrm{TI}^{\mathrm{n}}\right)$ index that considers direct and indirect effects spreading along pathways of up to $n$ step. This approach quantifies both the centrality of nodes in networks $\left(\mathrm{TI}_{\mathrm{i}}\right)$ and the strength of effects between node $i$ and node $j\left(\mathrm{Tl}_{\mathrm{ij}}\right)$. It is important to note that the latter can be calculated also for binary networks of un-weighted links: strength is the pure consequence of topology $[20,21]$.

The centrality of species (trophic groups) in food webs seems to be a systemic property with important ecological correlates (e.g. body size, mobility, see: [22]). There is a number of topological indices quantifying centrality in networks and their relationship is increasingly understood [23]. Since we must deal with indirect effects in order to assess the combinations of positive and negative impacts, we use the TI index, explicitly considering the length of direct and indirect pathways in food webs [20]. We note that $\mathrm{TI}$ is an useful centrality measure out of the many, according to a recent study wherer machine learning identified the most predictive combinations of centrality indices [24]. In this paper, we used $\mathrm{Tl}^{3}$, i.e. we considered indirect effects up to 3 steps. We performed these calculations in order to see whether there is structural basis of (constraints on) the sign-agreement (for nodes and networks) and signdeterminacy (for interactions and networks).

\section{Loop Analysis}

Loop analysis is a qualitative method, that uses signed digraphs to illustrate networks of interacting variables $[7,8]$. This technique gives the opportunity to represent the structure of linkages of the variables and the patterns of their variations $[25,26]$. Also, the qualitative models allow to examine the effect of non-biological variables on our system (such as gold mining, fishing etc.). This qualitative modeling approach provides a method that is useful where species and their natural history are well-known, but not quantified [27]. 
Signed digraphs are based on the generally accepted interactions between nodes (trophic groups). These interactions come from the previous trophic models in [13]. The figures show two types of connections: arrows $(\rightarrow)$ for positive and circle-head links $(-0)$ for negative effects. These links are originated from the coefficients of the community matrix [7]. The diagonal terms of the community matrices are self-effects on system variables, represented in signed digraphs as links connecting variables with themselves. These links are self-dampening (circle-headed) with self-limiting growth rate. For a simple prey-predator system, see Figure 1.

Using the qualitative modelling framework of loop analysis, one can analyze pathways and feedbacks in the system, making predictions about the response of variables to perturbations. These can be the addition (increased biomass) or deletion (decreased biomass) of other nodes. Based on feedbacks and pathways, one can qualitatively specify the direction of changes. If we analyse relatively small systems (with $\mathrm{n}<5$ nodes), this can be executed through the direct analysis of the signed digraph. For larger networks, the graphical feedback analysis is difficult but one can calculate response predictions from mathematical operations on the community matrix.

We followed the method described in [26] to get the predictions for our networks. The loop formula is used for calculating the equilibrium value of the variables following a perturbation, so it can be deduced how does the abundance of a certain variable change [28]:

$\frac{\delta x_{j}}{\delta c}=\frac{\sum_{i, k}\left\lfloor\frac{\delta f_{i}}{\delta c}\right\rfloor \times\left[p_{j i}^{(k)}\right] \times\left[F_{n-k}^{(c o m p)}\right]}{F_{n}}$

On the left side, $x_{j}$ is the variable with the equilibrium value being calculated and $c$ is the changing parameter (e.g. mortality, fecundity, abundance). On the right side, $f$ is the growth rate, $\partial f_{i} / \partial c$ designates whether the growth rate of the $i^{\text {th }}$ variable is increasing or decreasing (positive or negative input, respectively), $p_{j i}{ }^{(k)}$ is the pathway connecting the variable to the changed parameter (where the perturbation enters the system), $F_{n-k}\left(\right.$ comp) is the complementary feedback, which buffers or reverses the effects of the pathway and $F_{n}$ designates the overall feedback of the system, which is a measure of the inertia of the whole system to change [26, 28]. See also [8] for the discussion of the correspondence between matrix algebra and loop analysis.

The net effect (the sum of the direct and indirect effects) on variable $i$ resulting from a perturbation on variable $j$ is given by the $j-i^{\text {th }}$ element of the inverse community matrix $[A]^{-1}$. The sign of the coefficients of $[A]^{1}$ give the direction of the expected changes for the variables [26]. To make predictions, we used a routine that randomly assigns numerical values from an uniform distribution to the coefficients of the community matrix (these coefficients belongs to the links of the signed digraph). This was performed $100^{\star} N^{2}$ times, where $N$ is the number of variables in the system. Matrices satisfying the asymptotic Lyapunov criteria were accepted and inverted. The routine of [26] calculated predictions for the probabilities based on the percentage of positive and negative signs and zeroes in the inverted matrices. They defined a set of rules to make a final table of predictions only from signs (Table 2, [26]).

Table 2. The frequency of positive $\left({ }^{\circ}+\right)$ effects in the inverted matrices defines the sign of predictions.

\begin{tabular}{|ll|}
\hline$\%$ of " + " & sign \\
\hline $0-24$ & - \\
\hline $25-39$ & $?-$ \\
\hline $40-59$ & $0 *$ \\
\hline $60-74$ & $?+$ \\
\hline $75-100$ & + \\
\hline$\%$ of "0"=100 & 0 \\
\hline
\end{tabular}

Half of the effects correspond to clear predictions (lower 25\% for negative and top 25\% for positive prediction). Less than a third of the effects correspond to weak predictions (15-15\% for both weak negative and weak positive predictions). The fifth of the effects (the middle 20\%) correspond to net zero prediction ("0*"). Finally, „0" means no change in the abundance of the variable [26]. 
Community matrices show the links between the trophic groups. The elements in the rows affect the elements in the columns and the values could be $-1,0$ or 1 . These represent prey-predator (resouce-consumer) effects. We decided to work with self-dampening variables only for living groups (see Table 3 for the community matrix of site 1).

Table 3. Community matrix for site 1.

\begin{tabular}{|c|c|c|c|c|c|c|c|c|c|c|c|c|c|c|}
\hline & ALGA & CARN & COLF & COLG & DIAT & GRAZ & HEDE & HERB & LEAF & OMNI & РOM & PRED & SHRE & TERR \\
\hline ALGA & -1 & 0 & 0 & 0 & 0 & 1 & 1 & 1 & 0 & 1 & 0 & 0 & 0 & 0 \\
\hline CARN & 0 & -1 & -1 & -1 & 0 & -1 & -1 & -1 & 0 & -1 & 0 & -1 & -1 & -1 \\
\hline COLF & 0 & 1 & -1 & 0 & 0 & 0 & 0 & 0 & 0 & 1 & -1 & 1 & 0 & 0 \\
\hline COLG & 0 & 1 & 0 & -1 & 0 & 0 & 0 & 0 & 0 & 1 & -1 & 1 & 0 & 0 \\
\hline DIAT & 0 & 0 & 0 & 0 & -1 & 1 & 1 & 1 & 0 & 1 & 0 & 0 & 0 & 0 \\
\hline GRAZ & -1 & 1 & 0 & 0 & -1 & -1 & 0 & 0 & 0 & 1 & -1 & 1 & 0 & 0 \\
\hline HEDE & -1 & 1 & 0 & 0 & -1 & 0 & -1 & 0 & -1 & 0 & -1 & 0 & 0 & 0 \\
\hline HERB & -1 & 1 & 0 & 0 & -1 & 0 & 0 & -1 & 0 & 0 & -1 & 0 & 0 & 0 \\
\hline LEAF & 0 & 0 & 0 & 0 & 0 & 0 & 1 & 0 & 0 & 0 & 0 & 0 & 1 & 0 \\
\hline OMNI & -1 & 1 & -1 & -1 & -1 & -1 & 0 & 0 & 0 & -1 & -1 & -1 & -1 & -1 \\
\hline РОМ & 0 & 0 & 1 & 1 & 0 & 1 & 1 & 1 & 0 & 1 & 0 & 0 & 0 & 0 \\
\hline PRED & 0 & 1 & -1 & -1 & 0 & -1 & 0 & 0 & 0 & 1 & 0 & -1 & -1 & 0 \\
\hline SHRE & 0 & 1 & 0 & 0 & 0 & 0 & 0 & 0 & -1 & 1 & 0 & 1 & -1 & 0 \\
\hline TERR & 0 & 1 & 0 & 0 & 0 & 0 & 0 & 0 & 0 & 1 & 0 & 0 & 0 & 0 \\
\hline
\end{tabular}

Direct trophic interactions are represented in such a way that the elements in the rows affect the elements in the columns and the values could be -1, 0 or 1, depending on field data. We decided to work with self-dampening variables only for living groups.

We were interested in the effect of decreasing the biomass of each trophic group (i.e. the value of each variable), one by one. For this, we needed to simply reverse our predictions [29]. By predicting these changes, qualitative models can also predict the correlation patterns between the examined groups/variables $[8,26,28]$.

Levins' loop algorithm was extended in [29] to complex ecological systems. The adjoint of the negative of this community matrix shows the net number of complementary feedback cycles. A complementary feedback appears if $k$ variables in the path are ideally excluded from the graph: what remains is called the complementary subsystem. The complementary feedback is the feedback that groups all the variables in the complementary subsystem [26] that contribute to the responses of other variables in the whole system. Therefore, the adjoint of the ${ }^{\circ} \mathrm{A}$ is equivalent to Levins' loop analysis algorithm and its relation with the inverse matrix $\left(A^{-1}\right)$ is:

$-A^{-1}=\frac{\operatorname{adjoint}(-A)}{\operatorname{determinant}(-A)}$

where the adjoint and inverse matrices are calculated with the negative of the community matrix, thus the positive input is read down the columns and the responses along the rows [29].

An "absolute feedback” matrix was defined in [29] to calculate the absolute number of complementary feedback cycles in a response whether positive or negative:

$T_{i j}=$ permanent $\left(\operatorname{minor}^{\cdot} A_{i j}\right)^{T}$

Where ${ }^{`} A$ denotes the adjacency matrix (absolute values of ${ }^{\circ} A$ ). 
The „weighted-predictions” matrix can be calculated from the Eq. (1)-(2):

$$
W=\frac{\overrightarrow{\left|a d J^{\circ} A\right|}}{T}
$$

where " $\rightarrow$ " is a vectorised matrix operator, what denotes element-by-element division $\left(W_{i j}=1\right.$, when $\left.T_{i j}=0\right)$. The elements of $W$ show the probability of sign determinacy of response predictions in the adjoint matrix. If all of them are of the same sign in one cell, then $W_{i j}=1$. If there is an equal number of negative and positive feedback cycles, then $W_{i j}=0$ [29]. The $W_{i j}=0.5$ value is a threshold for sign determinacy in models of any size [27].

Our values in the "weighted predictions" matrix were mostly under $W_{i j}=0.5$. According to this, most of the prediction signs were indeterminate. We used the adjoint values to investigate if sign determinacy depends on the strength of interactions (based on both structure and simulations).

\section{Dynamical simulations}

For the dynamical simulation data on the food webs, we used earlier results of an individual-based model [13, 19]. This simulation model was built in a process algebra-based framework [30]: abundance was calculated for the trophic groups and interaction strength was converted to probability, according to the kinetics of the stochastic simulation framework used routinely in systems biology [31, $32,33,34]$.

The stochastic IBM simulation model, set up with the parameters descibed in the field, was balanced by a genetic algorithm. Following the reference runs, sensitivity analysis was performed. The abundance of each trophic group was perturbed and the response of each other trophic group was measured. Since this was a stochastic model, both the mean and the variability were evaluated.

In this paper, we used only the sign of the responses (not their strength). For comparability, minimal changes must be made in the results of the dynamical simulations: since these categories do not exist in simulations, ?+, ?- and 0* predictions were considered as +, - and 0 , respectively. Further, as the effects in dynamical simulations never result exactly in 0 , we needed to define zero effects during the simulations. We decided to have the same number of zeroes in the simulation results as we got in the predictions of loop analysis. Values in this "corridor" of the smallest positive and negative simulation outcomes were considered 0.

\section{Software}

We used MASS 7.3-51.5 and nlme 3.1-148 R packages for the analyses. For simulating sign predictions, we used the R code in [26]. The LoopAnalyst 1.2-6 package was used for the calculations of the adjoint of the - ${ }^{\circ} \mathrm{A}$ and the "weighted predictions" matrices (and it required the nIme package, too). Figure 3-4 were created with GVEdit Graph File Editor For Graphvizversion: 1.02 Graphviz version:2.38.

\section{Statistical methods}

Sign-agreement was examined on several levels. We compared the predictions of loop analysis to the calculations made by the structural importance index $\mathrm{Tl}^{3}$ and to the results of dynamical simulations for the whole networks, for individual nodes (i.e. the rows of the matrices) and for individual interactions.

As described above, we categorized the outcome of dynamical simulation results into 3 categories $(+, 0,-)$. This way, the table of predictions from loop analysis and the matrix of simulation effects became comparable (both containing only,+- and 0 ). A binary sign-agreement matrix contained 1 (yes) for similar and 0 (no) for different signs in the two matrices. The percentage of 1 values served for quantifying sign-agreement ( $1 \mathrm{~s}$ in the main diagonal, corresponding to self-effects, were not considered).

Chi-square tests were applied for the effect signs for each node, determining if there is significant difference in either loop analysis or dynamical simulations.

In order to see if there is some structural basis for sign-determinacy and sign-agreement, we tested the correlation between (1) the adjoint matrix and the dynamic simulation results, (2) the adjoint matrix and interaction strength calculated by $\mathrm{TI}_{\mathrm{ij}}$, (3) the dynamic 
simulation results and interaction strength calculated by $\mathrm{Tl}_{\mathrm{ij}}$ and (4) sign prediction of loop analysis and node centrality measured by $\mathrm{TI}_{i}$

\section{Results}

Figure 2 shows the food web for each of the 6 sites. The number of trophic groups varies between 12 and 15 , starting from 14 in the pristine forest (site 1).

The predicted sign of effects for each trophic group is shown in Figure 3, for each of the 6 sites (Figure 3a - 3f). In site 1 (Figure 3a), mutually negative (CARN-HEDE), mutually positive (CARN-OMNI), antagonistic (LEAF-GRAZ) uni-directional positive (TERR-PRED) and uni-directional negative (HERB-CARN) interactions can be seen. Between some pairs of trophic groups, there is no interaction predicted (e.g. CARN and COLG). Higher sign-agreement is seen for organisms at higher trophic levels. This pattern seems to be the same also for site 2 (Figure 3b) but not for the other sites. Site 4 (Figure 3d) is simpler than the previous sites, with some groups having only a single interaction partner (e.g. CARN, HEDE, COLG). The differences among the sites are quite characteristic in loop analysis, while the simulation models vary less. Site 6 (Figure $3 f$ ) is strongly dominated by factors essentially external to the stream ecosystem (TERR and HUMW).

Sign-agreement predicted by loop analysis and by the dynamic simulations are shown in Table 4, at the level of the whole networks and for individual nodes (this latter is represented by the colours in Figure 3: sign-agreement increases from light yellow to dark green). The mean correspondence of signs at the network level is under $50 \%$. Maximum sign-agreement ranged between 50 and $64.29 \%$ and the minimum was between 7.14 and $27.27 \%$. On average, site 6 in the Kelian River is the ecosystem where loop analysis best predicts the signs of dynamial simulation results. From site 2 to site 6 , there is a monotonous increase in mean (and the minimal) signagreement (the maximum values show no trend and also site 1 is out of this pattern). This suggests that the two methods provide different, complementary information on the ecosystem. Some organisms generally give consistent behaviour (e.g. HEDE), while others (ALGA, POM) behave differently in different modelling environments. Whether POM will have positive or negative impact on others is differently predicted in 5 out of 6 sites.

Table 4. Sign-agreement (between predictions of loop analysis and results of dynamical simulations) for nodes and networks.

\begin{tabular}{|llllll|}
\hline & Network min & Network mean & Network max & Node min & Node max \\
\hline site 1 & 23.08 & 38.46 & 61.54 & ALGA, COLF, POM, PRED & HEDE \\
\hline site 2 & 7.69 & 28.57 & 61.54 & ALGA, COLG, POM, SHRE & HEDE \\
\hline site 3 & 14.29 & 32.86 & 50 & SHRE & HEDE, CARN \\
\hline site 4 & 18.18 & 36.36 & 54.55 & ALGA, POM, OMNI & COLG, DIAT \\
\hline site 5 & 21.43 & 38.1 & 64.29 & ALGA, COLG, COLF, POM, TERR & PRED \\
\hline site 6 & 27.27 & 45.45 & 63.64 & POM & TERR \\
\hline
\end{tabular}

Mean, minimum and maximum values characterize whole matrices. The trophic groups with minimum (least predictable) and maximum (most predictable) values are shown.

Sign-agreement was measured also at the level of individual interactions. The mean of sign-agreement is also under $50 \%$ but the maximums are between $50-72 \%$ (Table 5). The high sign-agreement for HEDE is seen also in the interactions in this group, being involved in 4 out of 7 of the highest-agreement interactions. It is also noted that the same group (HEDE) can be involved in minimum (HEDE-POM) and maximum (HEDE-TERR) agreement interactions (see site 2). It is noted that COLG in site 5 has several minimalagreement interactions (e.g. COLG-POM), after being the most predictable group in site 4 (with the most predictable interaction, COLGCARN).

Table 5. Sign-agreement (between predictions of loop analysis and results of dynamical simulations) for interactions. 


\begin{tabular}{|c|c|c|c|c|c|}
\hline & Network min & Network mean & Network max & Interaction min & Interaction max \\
\hline $\begin{array}{l}\text { site } \\
1\end{array}$ & 19.23 & 35.71 & 65.38 & $\begin{array}{l}\text { ALGA-HERB, ALGA-SHRE, COLF-PRED, POM-CARN, } \\
\text { POM-GRAZ }\end{array}$ & HEDE-COLF \\
\hline $\begin{array}{l}\text { site } \\
2\end{array}$ & 3.85 & 26.53 & 50 & POM-HEDE & $\begin{array}{l}\text { HEDE-ALGA, HEDE- } \\
\text { TERR }\end{array}$ \\
\hline $\begin{array}{l}\text { site } \\
3\end{array}$ & 7.14 & 30.67 & 57.14 & COLF-FILA & HEDE-SHRE \\
\hline $\begin{array}{l}\text { site } \\
4\end{array}$ & 13.64 & 33.33 & 68.18 & $\begin{array}{l}\text { ALGA-HEDE, OMNI-HEDE, OMNI-TERR, POM- } \\
\text { HEDE, POM-TERR }\end{array}$ & COLG-CARN \\
\hline $\begin{array}{l}\text { site } \\
5\end{array}$ & 17.86 & 35.56 & 57.14 & $\begin{array}{l}\text { ALGA-COLG, CARN-COLG, COLF-COLG, POM- } \\
\text { COLG, TERR-COLG }\end{array}$ & PRED-LEAF \\
\hline $\begin{array}{l}\text { site } \\
6\end{array}$ & 18.18 & 41.67 & 68.18 & FILA-OMNI, POM-HERB & TERR-CARN \\
\hline
\end{tabular}

Mean, minimum and maximum values characterize whole matrices. The inter-specific interactions (direct and indirect effects) with minimum (least predictable) and maximum (most predictable) values are shown.

The Chi-square tests showed that the predictions of loop analysis for different trophic groups were significantly different only for site 4 of Kelian River (Figure 4a, see Appendix A for all of the other simulations), while the Chi-square tests for the results of dynamic simulations did not show significant differences (Figure 4b). Based on loop analysis (Figure 4a), CARN, COLG and HEDE have predominantly zero and occasionally positive effects on others. On the contrary, TERR (and to some extent POM) have the highest proportion of negative effects. Dynamical simulations (Figure 4b) show a different outcome with DIAT being the only one without negative effects and HEDE (and ALGA) with the largest negative impacts. According to Appendix A, there are significant differences among the distribution of impact signs of various organisms in almost no cases. In some cases, this homogeneity is quite strong (e.g. site 3, loop analysis with 3 categories), while there are models with non-significant but visible differences (e.g. GRAZ and HEDE in the simulation model of site 2). In the model of site 4, loop analysis with 5 sign categories also provided significant differences among the organisms: the pattern is qualitatively similar to the result of the model with 3 sign categories (see Figure 4).

Spearman correlation ( $|\rho|$ values) suggest no strong correlations between our values. Only the structural interaction strength $\left(\mathrm{TI}_{\mathrm{ij}}\right)$ and the adjoint values $\left(\mathrm{LA}_{\mathrm{adj}}\right)$ showed significant correlation in site 4 of the model (see Appendix $\mathrm{B}$ ).

\section{Conclusions}

Predictive food web research would be a quantitative and holistic toolkit for systems-based conservation efforts. Good predictions on strong and weak as well as positive and negative effects are important for management and policy. While dynamical simulation exercises require a large number of parameters and complicated models, the semi-quantitative methodology of loop analysis offers simpler and faster results, generally easier to understand and interpret. Structural analyses are the simplest and least realistic ones. The major question was to what extent simpler methods can replace the more complicated ones.

Comparing the sites, we see that the relatively consistent predictability of HEDE is seen in the sites of otherwise contrasting signs (site 2 , site 3). The most consistent interaction happens between TERR and CARN in the most consistent model of site 6 . This is the case when loop analysis and the dynamical simulation model mostly agree on sign prediction.

Based on Table 4, the general pattern emerges that higher sign-agreement is characteristic for trophic groups at higher trophic levels in sites 1-3, while trophic groups at lower trophic levels in sites 4-6.

Supposing that both models are relevant and correct, the question emerges why and to what extent should they be similar to each other. Similarity means that one reinforces the other, while differences may suggest that they are complementary, providing different kind of information. In the future, mixed trophic impact analysis, signed topological importance and further kinds of dynamical simulations must also be compared to the models studied here.

It is a question if experimental studies (e.g. mesocosm experiments) could help to better understand the predictability of effect signs and their differences in different modelling environments. Performing experiments only for a few species (e.g. perturbing COLG) might 
help a lot in calibrating the amount of expected changes and scale the models.

It needs to be better understand how trophic complexity is related to predictability, in terms of either interaction strengths or interaction signs. Earlier research, based on time series data, found quite convincing results on the dimensionality of niche space [35].

Future research can be extended, for example, towards (1) considering the asymmetry of interactions (MEPS) and (2) considering loop sign [36, 37]. The former could be important in order to better understand cause-effect relationships, as strong asymmetry may imply causality. The latter could help to better assess network-level stability.

\section{Abbreviations}

ALGA: green and blue-green algae

CARN: carnivorous fish

COLF: invertebrate collector-filterers

COLG: invertebrate collector-gatherers

DIAT: diatoms

FILA: filamentous bacteria

GRAZ: invertebrate grazers

HEDE: herbivore-detritivore fish

HERB: herbivorous fish

HUMW: human waste

LEAF: leaf litter

OMNI: omnivorous fish

POM: settled and suspended coarse and fine Particulate Organic Matter

PRED: invertebrate predators

SHRE: invertebrate shredders

TERR: terrestrial insects

\section{Declarations}

Ethics approval and consent to participate

Not applicable

Consent for publication

Not applicable

Availability of data and materials

The datasets used and/or analysed during the current study are available from the corresponding author on reasonable request.

Competing interests

The authors declare that they have no competing interests. 
Our research was supported by the National Research, Development and Innovation Office - NKFIH, grant GINOP-2.3.2-15-2016-00057. The authors acknowledge funding received from the European Union's Horizon 2020 research and innovation programme as part of the AtlantECO project under grant agreement No 862923. This output reflects only the author's view and the European Union cannot be held responsible for any use that may be made of the information contained therein.

\section{Authors' contributions}

V.F. performed the loop analysis modeling and the statistical analysis. She wrote the paper.

F.J. framed the research idea, coordinated the project and wrote the paper.

\section{Acknowledgments}

We are grateful to Catherine M. Yule for the data and earlier discussions on the database. We thank Sándor Imre Piross and István Reguly for earlier discussions on the methods.

\section{Acknowledgments}

We are grateful to Catherine M. Yule for the data and earlier discussions on the database. We thank Sándor Imre Piross and István Reguly for earlier discussions on the methods. Our research was supported by the National Research, Development and Innovation Office - NKFIH, grant GINOP-2.3.2-15-2016-00057. The authors acknowledge funding received from the European Union's Horizon 2020 research and innovation programme as part of the AtlantECO project under grant agreement No 862923. This output reflects only the author's view and the European Union cannot be held responsible for any use that may be made of the information contained therein.

\section{References}

1. Bertness, M. D., and Shumway, S. W.: Competition and facilitation in marsh plants. American Naturalist. 142, $718-724$ (1993)

2. Kareiva, P. M., and Bertness, M. D.: Re-examining the role of positive interactions in communities. Ecology. 78,1945 (1997)

3. Bruno, J. F., Stachowitz, J. J., and Bertness, M. D.: Inclusion of facilitation into ecological theory. Trends in Ecology and Evolution. $18,119-125$ (2003)

4. Ulanowicz, R.E.: Utricularia's secret: the advantage of positive feedback in oligotrophic environments. Ecological Modelling. 79, 4957 (1995)

5. Dong, X., Grimm, N.B., Heffernan, J.B., Muneepeerakul, R.: Interactions between physical template and self-organization shape plant dynamics in a stream ecosystem. Ecosystems. 23, 891-905 (2020)

6. Leemans, L., Martínez, I., van der Heide, T., van Katwijk, M. M., van Tussenbroek, B. I.: A mutualism between unattached coralline algae and seagrasses prevents overgrazing by sea turtles. Ecosystems. 23, 1631-1642 (2020)

7. Levins, R.: Qualitative analysis of partially specified systems. Annals New York Academy of Sciences. 231, 123-138 (1974)

8. Puccia, C. J., and Levins, R.: Qualitative modelling of complex systems: an introduction to loop analysis and time averaging. Cambridge, Mass.: Harvard University Press (1985)

9. Ulanowicz RE, Puccia CJ.: Mixed trophic impacts in ecosystems. Coenoses. 5, 7-16 (1990)

10. Bondavalli, C., Ulanowicz, R.E.: Unexpected effects of predators upon their prey: the case of the American alligator. Ecosystems. 2: 49-63 (1999)

11. Liu, W. C., Chen, H. W., Jordán, F., Lin, W. H., and Liu, W. J.: Quantifying the interaction structure and the topological importance of species in food webs: a signed digraph approach. Journal of Theoretical Biology. 267, 355-362 (2010)

12. Liu, W. C., Huang, L. C., Liu, C. W., and Jordán, F.: A simple approach for quantifying node centrality in signed and directed social networks. Applied Network Science. 5, 46 (2020)

13. Jordán, F., Gjata, N., Mei, S., and Yule, C. M.: Simulating food web dynamics along a gradient: Quantifying human influence. PLoS ONE 7, e40280 (2012) 
14. Ortiz, M., Levins, R., Campos, L., Berrios, F., Campos, F., Jordán, F., Hermosillo, B., Gonzalez, J., and Rodriguez, F.: Identifying keystone trophic groups in benthic ecosystems: implications for fisheries management. Ecological Indicators. 25, 133-140 (2013)

15. Ortiz, M., Rodriguez-Zaragosa, F., Hermosillo-Nunez, B., and Jordán, F.: Control strategy scenarios for the alien lionfish Pterois volitans in Chinchorro Bank (Mexican Caribbean) based on semi-quantitative loop network analysis. PloS ONE 10, e0130261 (2015)

16. Ortiz, M., Hermosillo-Nuñez, B., González, J., Rodríguez-Zaragoza, F., Gómez, I., and Jordán, F.: Quantifying keystone species complexes: ecosystem-based conservation management in the King George Island (Antarctic Peninsula). Ecological Indicators. 81, 453-460 (2017)

17. Yule, C. M.: The impact of sediment pollution on the benthic invertebrate fauna of the Kelian River, East Kalimantan, Indonesia. Tropical Limnology. 3, 61-75 (1995)

18. Yule, C. M., Boyero, L., and Marchant, R.: Effects of sediment pollution on food webs in a tropical river (Borneo, Indonesia). Marine and Freshwater Research. 61, 204-213 (2010)

19. Jordán, F., Scotti, M., and Yule, C. M.: Food web simulations: stochastic variability and systems-based conservation. In: Adaptive Food Webs. Moore, J.C., de Ruiter, P.C., McCann, K.S. and Wolters, V. (Eds.), Cambridge University Press. 342-351 (2017)

20. Jordán, F., Liu, W.-C. and van Veen, F.J.F.: Quantifying the importance of species and their interactions in a host-parasitoid community. Community Ecology. 4, 79-88 . (2003)

21. Jordán, F.: Children in time: community organisation in social and ecological systems. Current Science. 97, 1579-1585 (2009)

22. Olmo Gilabert, R., Navia, A.F., De La Cruz-Agüero, G., Molinero, J.C., Sommer, U., Scotti, M.: Body size and mobility explain species centralities in the Gulf of California food web. Community Ecology. 20, 149-160 (2019)

23. Scotti, M., Podani, J., Jordán, F.: Weighting, scale dependence and indirect effects in ecological networks: A comparative study. Ecological Complexity. 4(3), 148-159 (2007)

24. Gouveia, C., Móréh, Á., Jordán, F.: Combining centrality indices: maximizing the predictability of keystone species in food webs. Ecological Indicators. in press.

25. Dambacher, J. M., Ramos-Jiliberto, R.: Understanding and predicting effects of modified interactions through a qualitative analysis of community structure. The Quarterly Review of Biology. (2007)

26. Bodini, A., and Clerici, N.: Vegetation, herbivores and fires in savanna ecosystems: A network perspective. Ecological Complexity. 28, 36-46 (2016)

27. Dambacher, J. M., Li, H. W., and Rossignol, P. A.: Qualitative predictions in model ecosystems, Ecological Modelling. 161, 79-93 (2003)

28. Bodini, A.: Reconstructing trophic interactions as a tool for understanding and managing ecosystems: application to a shallow eutrophic lake. Canadian Journal of Fisheries and Aquatic Sciences. 57, 1999-2009 (2000)

29. Dambacher, J. M., Li, H. W., Rossignol, P. A.: Relevance of community structure in assessing indeterminacy of ecological predictions. Ecology. 83, 1372-1385 (2002)

30. Jordán, F., Scotti, M. and Priami, C.: Process algebra-based models in systems ecology. Ecological Complexity. 8, 357-363 (2011)

31. Gillespie, D.T.: Exact stochastic simulation of coupled chemical reactions. J. Phys. Chem. 81, 2340-2361 (1977)

32. Priami, C., Quaglia, P.: Modelling the dynamics of biosystems. Brief Bioinform. 5, 259-269 (2004)

33. Dematté, L., Priami, C., Romanel, A., Soyer, O.: Evolving BlenX programs to simulate the evolution of biological networks. Theor. Comp. Sci. 408, 83-96 (2008)

34. Priami, C.: Algorithmic systems biology. Commun. ACM. 52, 80-89 (2009)

35. Stenseth, N.C., Falck, W., Bjørnstad, O.N., Krebs, C.J.: Population regulation in snowshoe hare and Canadian lynx: Asymmetric food web configurations between hare and lynx. Proceedings of the National Academy of Sciences. 94, 5147-5152 (1997)

36. Harary, F.: Status and contrastatus. Sociometry. 22, 23-43 (1959)

37. Wey, T., Jordán, F., and Blumstein, D.: Transitivity and structural balance in animal social networks. Behav. Ecol. Sociobiol. 73, 88 (2019)

38. Neuwirth, E., \& Brewer, R. C.: ColorBrewer palettes. R package version, 1. (2014)

\section{Figures}




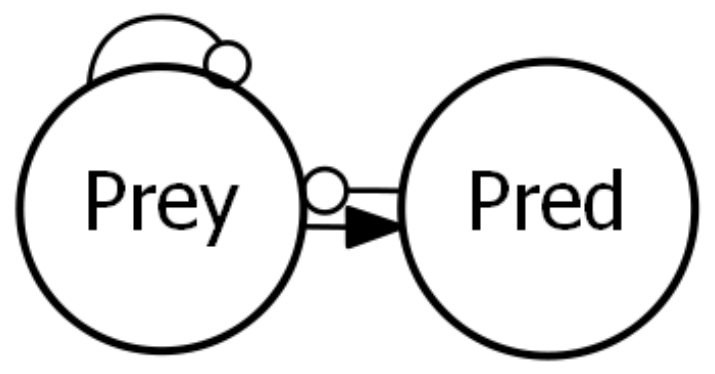

Figure 1

Signed digraph of a simple prey-predator system. The prey affects positively the predator („Pred”), indicated by a black arrow. Empty circles indicate negative effects: from the predator to the prey and the self-effect of the prey. 
b
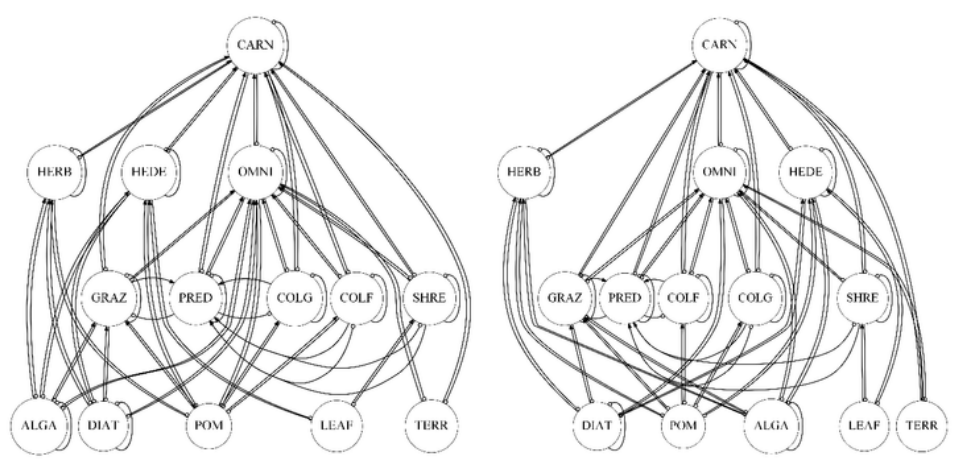

c

d
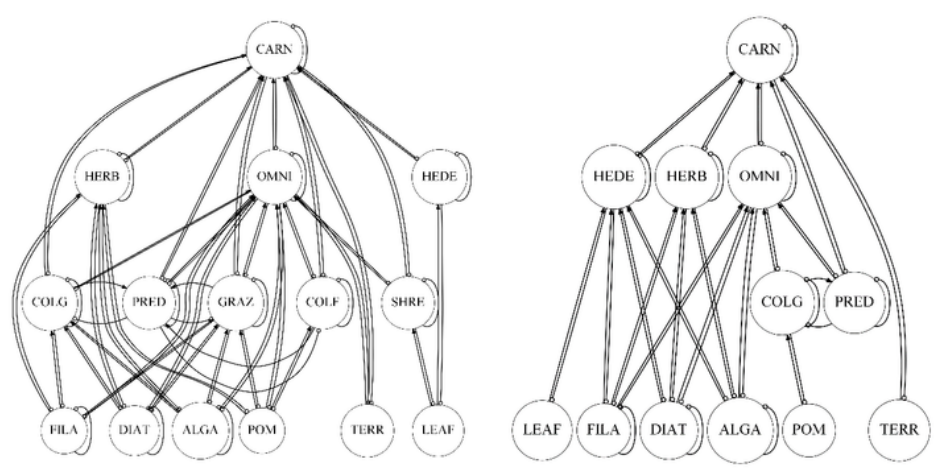

f
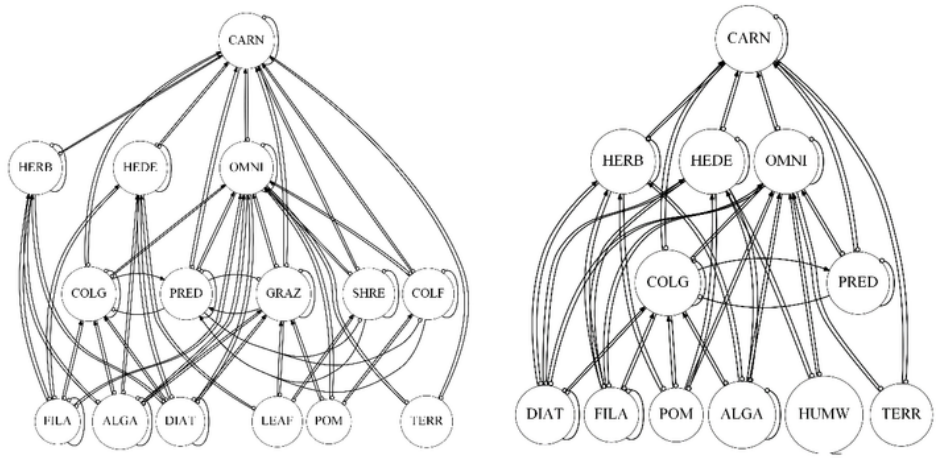

Figure 2

Food web of the Kelian River ecosystem in sites 1-6 (a-f). The arrow shows the consumer and the circle shows the resource for each trophic flow (who eats whom). Trophic groups are arranged according to trophic height. 


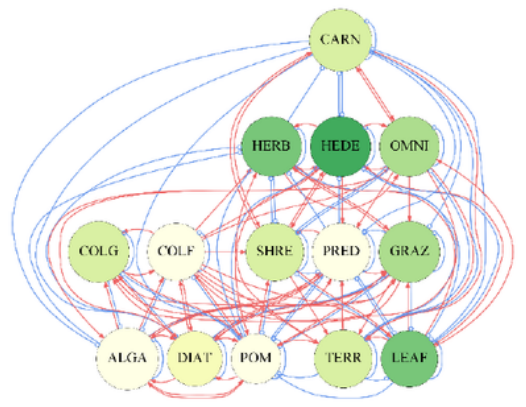

c
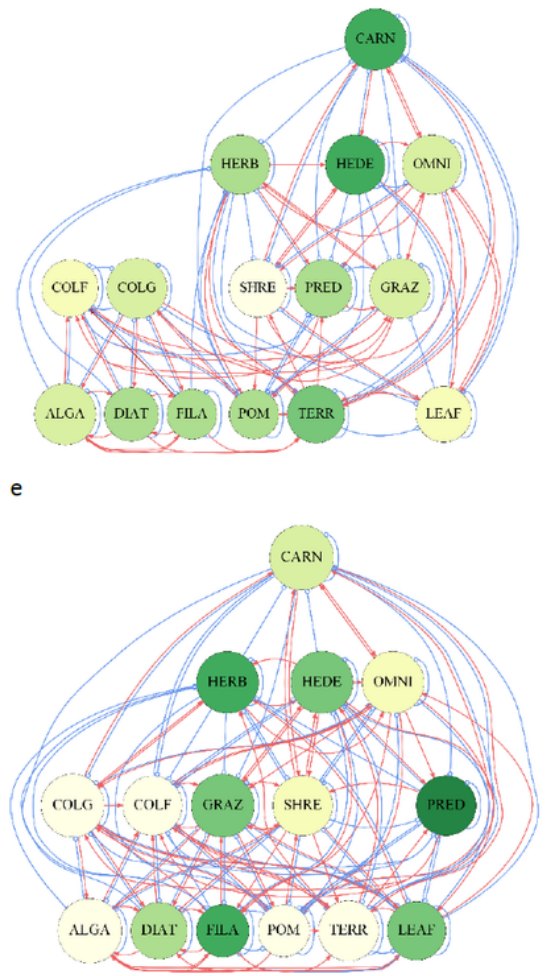

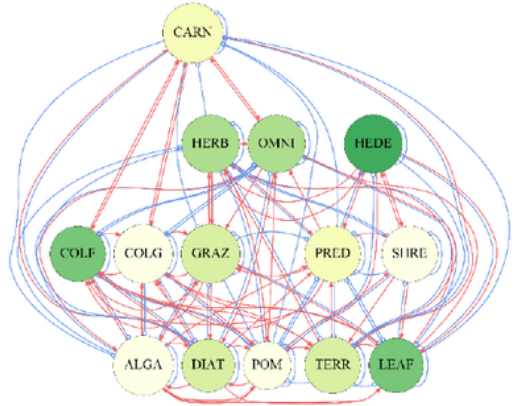

d
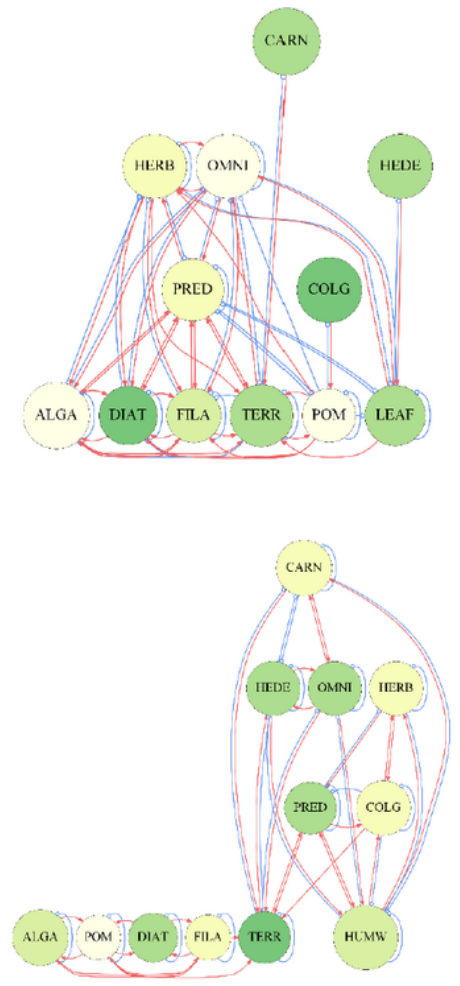

\section{Figure 3}

Food web of the Kelian River ecosystem in sites 1-6 (a-f). Arrows show the predictions of loop analysis: the sign of the effect of decreasing the abundance of a node on another (red is positive, blue is negative). The colour of nodes shows sign-agreement (\%) between the predictions of loop analysis and the results of dynamical simulations (increasing from light yellow to dark green). Whether perturbing the light yellow nodes has positive or negative effect on others largely depends on the model chosen (see Table X). On the contrary, the perturbation of dark green nodes is similarly predicted by both approaches (see Table X). Colored by ColorBrewer [38]. 

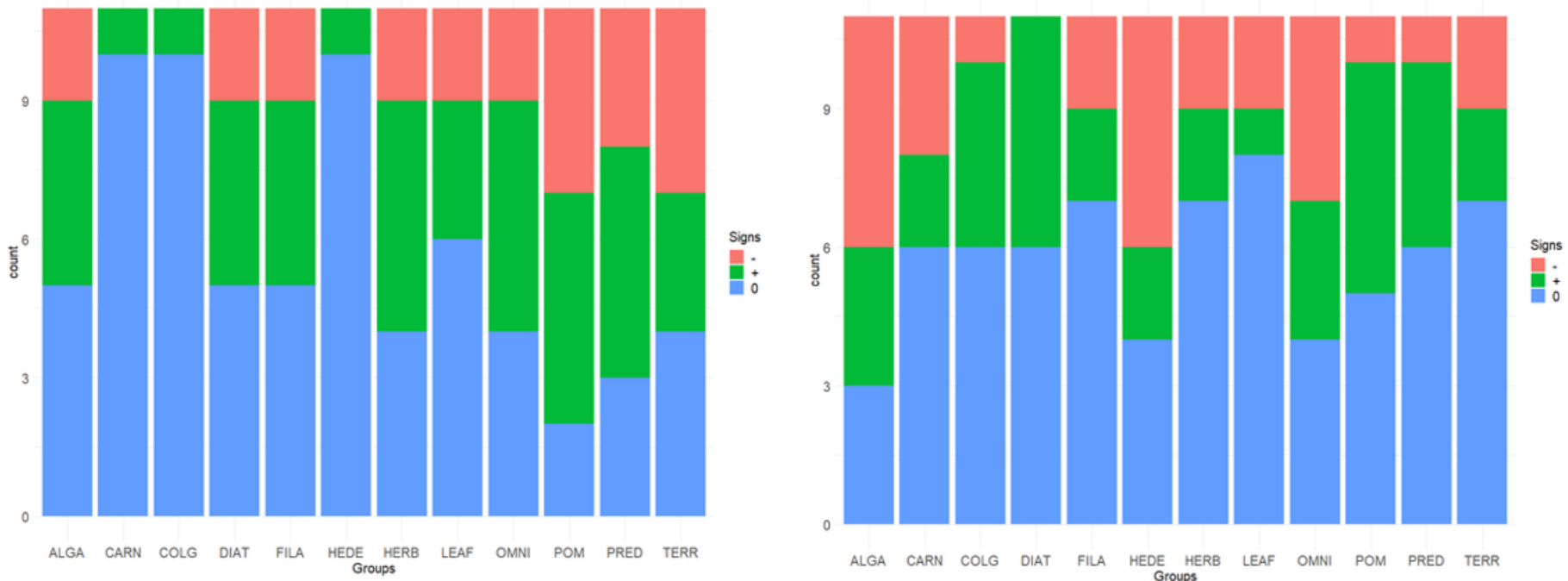

\section{Figure 4}

The number of positive (green), negative (red) and zero (blue) effects. Each 14 functional group are listed along the $\mathrm{x}$ axis, and the 13 other are counted along the $y$ axis. This figure represents site 4 of the Kelian River ecosystem model, based on loop analysis (a) and dynamical simulations (b). In order to make them comparable, the predictions of loop analysis were transformed: we used only 3 categories, + ? was replaced by,+- ? was replaced by - and $0 *$ was replaced by 0 . The sign composition of organisms differs

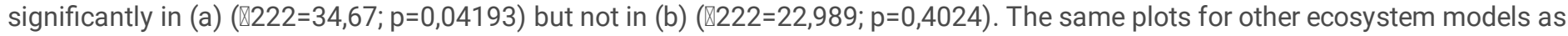
well as the plots for loop analysis with 6 prediction categories are shown in Appendix A.

\section{Supplementary Files}

This is a list of supplementary files associated with this preprint. Click to download.

- AppendixA.xlsx

- AppendixB.xlsx 